\title{
Tar Dosage Form
}

National Cancer Institute

\section{Source}

National Cancer Institute. Tar Dosage Form. NCI Thesaurus. Code C78749.

A semi-solid that is oily and viscous and composed of a hydrocarbon based resin formed from the breakdown and distillation of org anic substances. 